\title{
Evacuation gastrique et composition en acides aminés des protéines du régime chez le porc
}

\author{
J.P. LAPLACE, Odile PONS *, J.C. CUBER et Chantal KABORE \\ avec la collaboration technique de Régine Calmes, Claire Chabanet*, \\ Brigitte Mourot et A. Van Hecke \\ I.N.R.A., Laboratoire de Physiologie de la Nutrition, \\ * Laboratoire de Biométrie, Centre de Recherches zootechniques, \\ F 78350 Jouy-en-Josas
}

\begin{abstract}
Résumé
Cinq porcs de race Large White, mâles castrés, d'un poids vif moyen de $52,5 \mathrm{~kg}$, porteurs d'une canule gastrique permanente, sont utilisés pour la mesure des quantités de matière fraîche, matière sèche, amidon et azote, résiduelles dans l'estomac à divers temps $(30 \mathrm{mn}, 1 \mathrm{~h}, 2 \mathrm{~h}, 4 \mathrm{~h}$ et $7 \mathrm{~h})$ après ingestion d'un régime à base d'amidon de blé purifié dont les protéines sont apportées par de la caséine. Deux cinétiques sont établies pour chacun des 5 porcs. Ces observations sont comparées à celles obtenues antérieurement dans des conditions identiques pour des régimes à base du même amidon de blé, associé selon le cas à un gluten de blé ou une farine de poisson, compte tenu des compositions respectives en acides aminés de ces 3 protéines.

L'étude de la décroissance des quantités résiduelles dans l'estomac montre une grande similitude pour les régimes caséine et poisson, les quantités recueillies étant significativement plus importantes dans le cas du gluten 4 et $7 \mathrm{~h}$ après le repas. Ces différences liées au régime ne sont pas significatives au terme d'une analyse multivariate portant sur les quantités évacuées, alors que l'interférence de facteurs temporels ou liés à l'animal s'avère importante. Mais l'estimation de l'effet fixe du régime fournit des courbes d'évacuation qui corroborent les observations faites sur les quantités résiduelles. Il apparaît ainsi que la nature des protéines alimentaires conduit, pour les cas considérés, à deux types d'effets. L'évacuation gastrique de l'azote total est influencée, surtout au cours des premières heures postprandiales, par la proportion d'azote soluble dans l'aliment : elle est donc plus importante pour l'aliment poisson que pour les 2 autres régimes. Mais, globalement, l'évacuation de la matière sèche est analogue pour les régimes caséine et poisson dont les compositions en acides aminés présentent une grande similitude; elle est plus lente pour le régime gluten dont la composition en acides aminés est fort différente.
\end{abstract}

Mots clés : Evacuation gastrique, porc, acides aminés, digestion, protéines alimentaires.

\section{Introduction}

Il est couramment admis que la matière sèche des régimes protéiprives fait, chez le porc, l'objet d'une évacuation gastrique globalement plus rapide que celle des régimes azotés au cours des premières heures postprandiales (RERAT \& Lougnon, 
1963 ; Zebrowska \& Buraczewska, 1972 ; Zebrowska et al., 1975). Pourtant, durant les 2 premières heures après le repas, la matière sèche d'un régime à 16 p. 100 de protéines apportées par du gluten de blé est évacuée à un rythme analogue à celui qui est constaté pour un régime protéiprive (LAPlace et al., 1981). Cette même rapidité relative d'évacuation a été hypothétiquement attribuée à une absence d'inhibition à point de départ duodénal, du fait du défaut de protéines dans le cas d'un aliment protéiprive. On peut aussi concevoir que des protéines de configuration complexe soient hydrolysées relativement lentement dans l'intestin grêle proximal, avec pour conséquence une faible inhibition d'origine duodénale. Tel pourrait être le cas pour les protéines du gluten de blé en raison d'une teneur relativement élevée en proline par rapport à une farine de poisson par exemple (Henry, PION \& Rerat, 1976). Toutefois, pour des temps postprandiaux plus importants, l'évacuation de la matière sèche d'un régime comportant du gluten apparaît à l'inverse nettement plus lente (ZEBrowSKA, 1973 a ; LAPlaCE et al., 1981). On note aussi, parmi les régimes testés par ZEBRowSKa (1973 a), une évacuation plus lente (en 8 h) de la matière sèche des aliments comportant de la caséine ou du gluten de blé, par rapport à ceux qui contiennent du soja ou de la féverole. Or, selon ZeBrowska (1973 b) caséine et gluten de blé présentent des taux de proline comparables, nettement supérieurs à ceux des autres sources protéiques.

Ces observations nous ont incités à quantifier l'évacuation gastrique d’un aliment dont les protéines sont apportées par de la caséine, puis à tenter la comparaison de ces résultats à ceux précédemment obtenus (LAPlace et al., 1981 ; LAPLACE, Pons \& Simon, 1983) pour des régimes comportant, selon le cas, du gluten de blé ou de la farine de poisson, compte tenu de la composition en acides aminés de ces trois régimes.

\section{Matériel et méthodes}

\section{A. Animaux et aliments}

Cinq porcs de race Large White, mâles castrés, d'un poids vif initial de $52,5 \pm$ $0,3 \mathrm{~kg}$ (moyenne \pm écart-type de la moyenne) ont été utilisés dans des conditions antérieurement définies (Cuber, Laplace \& Villiers, 1980). Sous anesthésie générale à l'halothane, on réalise une laparotomie sur la ligne médio ventrale, entre l'appendice xyphoïde et l'ombilic. Une canule en vinyle (diamètre int./ext. 22-30 mm) est mise en place après gastrotomie à mi-hauteur de la grande courbure de l'estomac. Elle est extériorisée, par la plaie opératoire, juste en arrière de l'appendice xyphoïde. Les porcs sont alors installés individuellement en cage à métabolisme et habitués à l'aliment expérimental durant 8 jours avant toute mesure.

L'aliment expérimental, dit «caséine», est à base d'amidon de blé purifié. Il est fabriqué manuellement pour un taux de matières azotées totales de 14 p. 100 et un taux de cellulose de bois purifiée de 6 p. 100. La composition exacte de cet aliment «caséine» est indiquée dans le tableau 1, comparativement à celle des aliments dits «poisson» et "gluten》 antérieurement utilisés (LAPLACE et al., 1981). Leurs compositions respectives en acides aminés sont détaillées dans le tableau 2. 


\section{TABleaU 1}

Composition respective des régimes et quantités réellement ingérées de matière sèche (MS), amidon et azote à l'occasion des repas d'épreave.

Respective composition of the diets, and quantities of dry matter (DM) starch and nitrogen really eaten at each test meal.

\begin{tabular}{|c|c|c|c|}
\hline $\begin{array}{c}\text { Aliment } \\
\text { Diet }\end{array}$ & $\begin{array}{l}\text { Caséine } \\
\text { Casein }\end{array}$ & $\begin{array}{l}\text { Gluten } \\
\text { Wheat gluten }\end{array}$ & $\begin{array}{c}\text { Poisson } \\
\text { Fish meal }\end{array}$ \\
\hline \multicolumn{4}{|l|}{$\begin{array}{l}\text { Constituants des alimerts (p. 100) } \\
\text { Feed constituents (per cent) }\end{array}$} \\
\hline $\begin{array}{l}\text { Amidon de blé } \ldots \ldots \ldots \ldots \ldots \ldots \ldots \\
\text { Wheat starch }\end{array}$ & 72,3 & 72,69 & 68,26 \\
\hline $\begin{array}{l}\text { Caséine chlorhydrique } \ldots \ldots \ldots \ldots \ldots \ldots \\
\text { Hydrochloric casein }\end{array}$ & 16,1 & - & - \\
\hline $\begin{array}{l}\text { Gluten de blé } \ldots \ldots \ldots \ldots \ldots \ldots \ldots \ldots \\
\text { Wheat gluten }\end{array}$ & - & 17,48 & - \\
\hline $\begin{array}{l}\text { Farine de poisson } \ldots \ldots \ldots \ldots \ldots \ldots \ldots \\
\text { Fish meal }\end{array}$ & - & - & 21,65 \\
\hline $\begin{array}{l}\text { Cellulose } \\
\text { Cellukse }\end{array}$ & 6,0 & 5,90 & 6,08 \\
\hline $\begin{array}{l}\text { Huile darachide } \ldots \ldots \ldots \ldots \ldots \ldots \ldots \\
\text { Peanut oil }\end{array}$ & 2,0 & 一 & - \\
\hline & 2,5 & 2,95 & 3,01 \\
\hline & 1,0 & 0,98 & 1,00 \\
\hline $\begin{array}{l}\text { Anti oxydant } \ldots \ldots \ldots \ldots \ldots \ldots \ldots \ldots \\
\text { Anti oxydant }\end{array}$ & 0,1 & - & - \\
\hline \multicolumn{4}{|l|}{$\begin{array}{l}\text { Composition chimique } \\
\text { Chemical composition }\end{array}$} \\
\hline $\begin{array}{l}\text { Azote }(1) \ldots \ldots \ldots \ldots \ldots \ldots \ldots \ldots \ldots \\
\text { Nitrogen }\end{array}$ & 2,2 & 2,5 & 2,7 \\
\hline $\begin{array}{l}\text { Amidon (1) } \ldots \ldots \ldots \ldots \ldots \ldots \ldots \ldots \\
\text { Starch }\end{array}$ & 70.0 & 74,0 & 69,0 \\
\hline $\begin{array}{l}\text { Energie brute (2) ....... } \\
\text { Crude energy }\end{array}$ & 4463 & 4286 & 4217 \\
\hline \multicolumn{4}{|l|}{$\begin{array}{l}\text { Repas d'épreuve ingéré } \\
\text { Test meal intake }\end{array}$} \\
\hline $\begin{array}{l}\text { Matière sèche }(g) \ldots \ldots \ldots \ldots \ldots \ldots \ldots \\
\text { Dry matter }\end{array}$ & 872 & 880 & 880 \\
\hline $\begin{array}{l}\text { Amidon }(\mathrm{g}) \ldots \ldots \ldots \ldots \ldots \ldots \ldots \ldots \\
\text { Starch }\end{array}$ & 610 & 651 & 607 \\
\hline $\begin{array}{l}\text { Azote }(\mathrm{g}) \ldots \ldots \ldots \ldots \ldots \ldots \ldots \ldots \\
\text { Nitrogen }\end{array}$ & 19,3 & 21,6 & 23,8 \\
\hline
\end{tabular}

(1) En p. 100 de la MS. As per cent of $D M$.

(2) En $\mathrm{kcal}$ par $\mathrm{kg}$ de MS, détermination sur 3 échantillons pour chaque régime. As kcal per kg DM, mean value for 3 samples from each diet. 


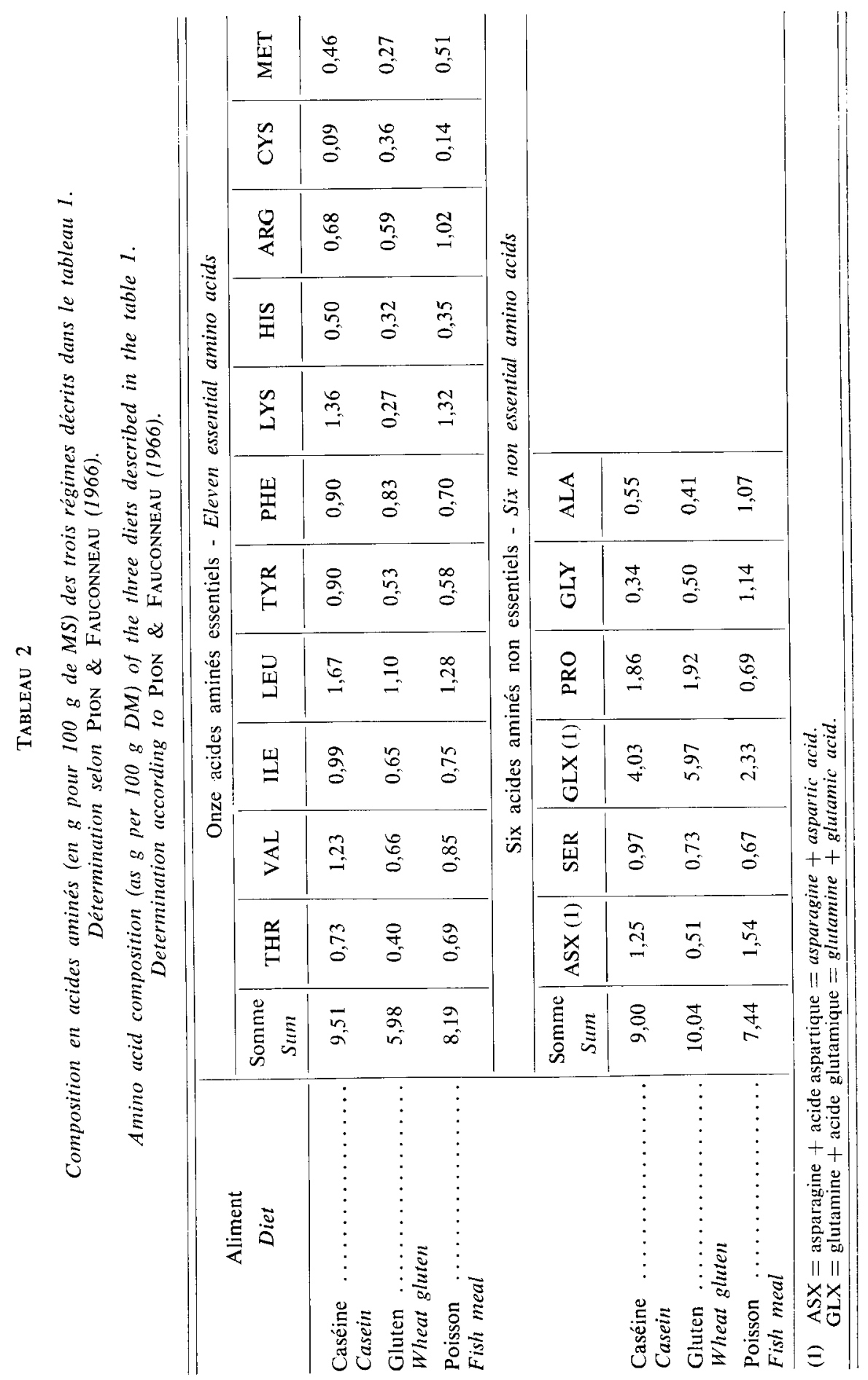


L'aliment caséine est systématiquement distribué en 2 repas quotidiens, à 9 et

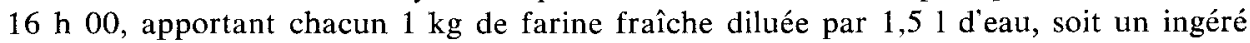
sec de $872 \mathrm{~g}$ pour chaque repas (tabl. 1). L'eau de boisson est apportée en totalité par l'eau de dilution de l'aliment en l'absence de tout abreuvement complémentaire.

\section{B. Collecte des digesta et méthodes analytiques}

La collecte des contenus gastriques est pratiquée selon une méthodologie décrite antérieurement (Cuber, Laplace \& Villiers, 1980), dans tous les cas à la suite du repas du matin. Avant le repas $(9 \mathrm{~h} 00)$, on procède au contrôle habituel de la vacuité gastrique. Une seule collecte des contenus gastriques résiduels est effectuée chaque jour, $30 \mathrm{mn}, 1 \mathrm{~h}, 2 \mathrm{~h}, 4 \mathrm{~h}$ ou $7 \mathrm{~h}$ après le repas d'épreuve. Cette collecte peut être considérée comme totale, ainsi qu'il a été vérifié par rinçage de l'estomac et par abattage immédiatement après la collecte. Le porc reçoit, aussitôt après la collecte, un repas de remplacement équivalant à la quantité collectée (sur la base d'une teneur moyenne en matière sèche de $30 \mathrm{p}$. 100). Lorsque la collecte a lieu $7 \mathrm{~h}$ après le repas d'épreuve, le repas de remplacement est fourni en complément du second repas quotidien. Chacun des 5 porcs est utilisé 10 fois, à raison de 2 mesures pour chacun des 5 instants d'observation, de façon à constituer 2 «cinétiques » par porc. Chaque cinétique est constituée de façon complète en une semaine. Les animaux sont sacrifiés, 24 jours après l'opération, à un poids vif moyen de $62,8 \pm 0,5 \mathrm{~kg}$.

Les contenus gastriques résiduels sont pesés, homogénéisés et échantillonnés en vue de la détermination de la teneur en matière sèche $\left(24 \mathrm{~h}\right.$ à l'étuve à $\left.104{ }^{\circ} \mathrm{C}\right)$ d'une part, et après lyophilisation, des teneurs en azote total (méthode Kjeldahl) et en amidon d'autre part. L'amidon est défini comme l'ensemble du glucose et de tous ses polymères alpha, et dosé selon CubER \& LAPLACE (1979). On peut alors calculer les quantités totales de matière sèche, amidon et azote, résiduelles dans l'estomac aux divers temps de collecte.

\section{Analyse statistique}

Les quantités moyennes résiduelles de matière fraîche, matière sèche, amidon et azote, exprimées en pourcentages des quantités ingérées correspondantes, sont comparées (test de Student) pour chaque instant de mesure aux quantités moyennes homologues enregistrées pour les aliments poisson et gluten (LAPLACE et al., 1981).

Au-delà de cette première approche, une analyse des effets susceptibles de rendre compte des différences d'évacuation gastrique a été conduite sur les quantités évacuées ( $Q$ ingérée - $Q$ résiduelle) exprimées en grammes. Toutefois, en raison des différences entre les protocoles de la présente étude et de l'expérience antérieure (LAPLACE et al., 1981), l'analyse de variance ne porte ici que sur 3 porcs $\times 2$ cinétiques pour chacun des régimes caséine, poisson et gluten. Pour ces derniers, on a retenu les deux premières des 3 cinétiques établies chez 3 porcs. Pour la caséine, on a retenu les deux cinétiques de 3 porcs choisis au hasard parmi les 5 utilisés pour les mesures. Le nombre d'observations disponibles pour l'analyse des effets est donc limité à 6 par instant et par régime.

Cette analyse de variance est conduite selon la méthodologie décrite antérieurement (Laplace, Pons \& Simon, 1983). Le test des liens entre les mesures effectuées 
aux différents instants (test de la matrice de variance-covariance) fournit, pour les quatre variables étudiées, des valeurs de la statistique du rapport de vraisemblance conduisant (selon une loi de $\chi^{2}$ ) à rejeter l'hypothèse d’indépendance des données. Aussi est-il nécessaire d'utiliser un modèle multivariate constitué par 18 vecteurs données ( 3 régimes $\times 3$ porcs $\times 2$ cinétiques) de taille 5 , c'est-à-dire comportant chacun les 5 mesures effectuées sur un porc pour constituer une cinétique en une semaine. La durée constitue donc une dimension du modèle et ne peut être prise en compte comme un facteur.

Le modèle retenu s'écrit :

$$
\overrightarrow{\mathrm{Y}_{\mathrm{rps}}}=\left(\mathrm{a}+\mathrm{b}_{\mathrm{r}}+\mathrm{I}_{\mathrm{rp}}+\mathrm{c}_{\mathrm{s}}+\mathrm{d}_{\mathrm{rs}}+\mathrm{J}_{\mathrm{rps}}\right) 1, \ldots, 5
$$

Par rapport à une valeur moyenne (terme a), l'addition du terme $b_{r}$ introduit l'effet propre du régime. Le terme $\mathrm{I}_{\mathrm{rp}}$ rend compte de l'effet hiérarchique régime/ porc dans la mesure où, chaque animal étant assigné à un seul régime, l'effet aléatoire du porc est lié à l'effet du régime. Le terme $\mathrm{c}_{\mathrm{s}}$ exprime l'effet de la semaine de constitution d'une cinétique; en effet, si l'indépendance des mesures d'une semaine à l'autre est admise a priori, les cinétiques consécutives ne sont pas nécessairement d'authentiques répétitions. Le terme $\mathrm{d}_{\mathrm{rss}}$ correspond à l'interaction régime-semaine. La résiduelle $J_{\mathrm{rps}}$ introduit toute autre source de variation non contrôlée.

Pour chacune des variables étudiées et chacun des termes du modèle, 1hypothèse de nullité du facteur considéré est testée par une statistique qui suit une loi de Wilks. Le calcul permet d'obtenir une estimation des divers termes à partir de laquelle peuvent être construites des courbes moyennes d'évacuation exprimant tel ou tel des effets fixes du modèle. Eventuellement, l'influence du temps peut être approchée en procédant à l'étude de ces courbes par application du modèle de PotThofF \& RoY (1964) pour choisir le degré du polynôme le mieux adapté pour décrire l'évacuation gastrique en fonction du temps.

\section{Résultats}

\section{A. Quantités résiduelles dans l'estomac}

L'évolution, au cours des 7 heures après ingestion de l'aliment caséine, des quantités résiduelles de matière fraîche, matière sèche, amidon et azote dans l'estomac, est décrite par la figure 1, comparativement à celle antérieurement enregistrée pour les aliments poisson et gluten.

La quantité de matière fraîche résiduelle, après ingestion de l'aliment caséine, ne diffère jamais significativement de celle observée pour l'aliment poisson ; elle est légèrement supérieure $(\mathrm{P}<0,10)$ à celle mesurée pour l'aliment gluten aux temps $1 \mathrm{~h}$ et $2 \mathrm{~h}$, mais lui est nettement inférieure $(P<0,05)$ au temps $7 \mathrm{~h}$.

Dans le cas de la matière sèche, les quantités résiduelles, après ingestion des aliments caséine et poisson, évoluent de façon analogue quoiqu'elles diffèrent entre elles significativement à $1 \mathrm{~h}(\mathrm{P}<0,05), 2$ et $4 \mathrm{~h}(\mathrm{P}<0,10)$ en raison de la très faible variabilité des mesures. Mais, pour ces deux régimes caséine et poisson, les quantités 

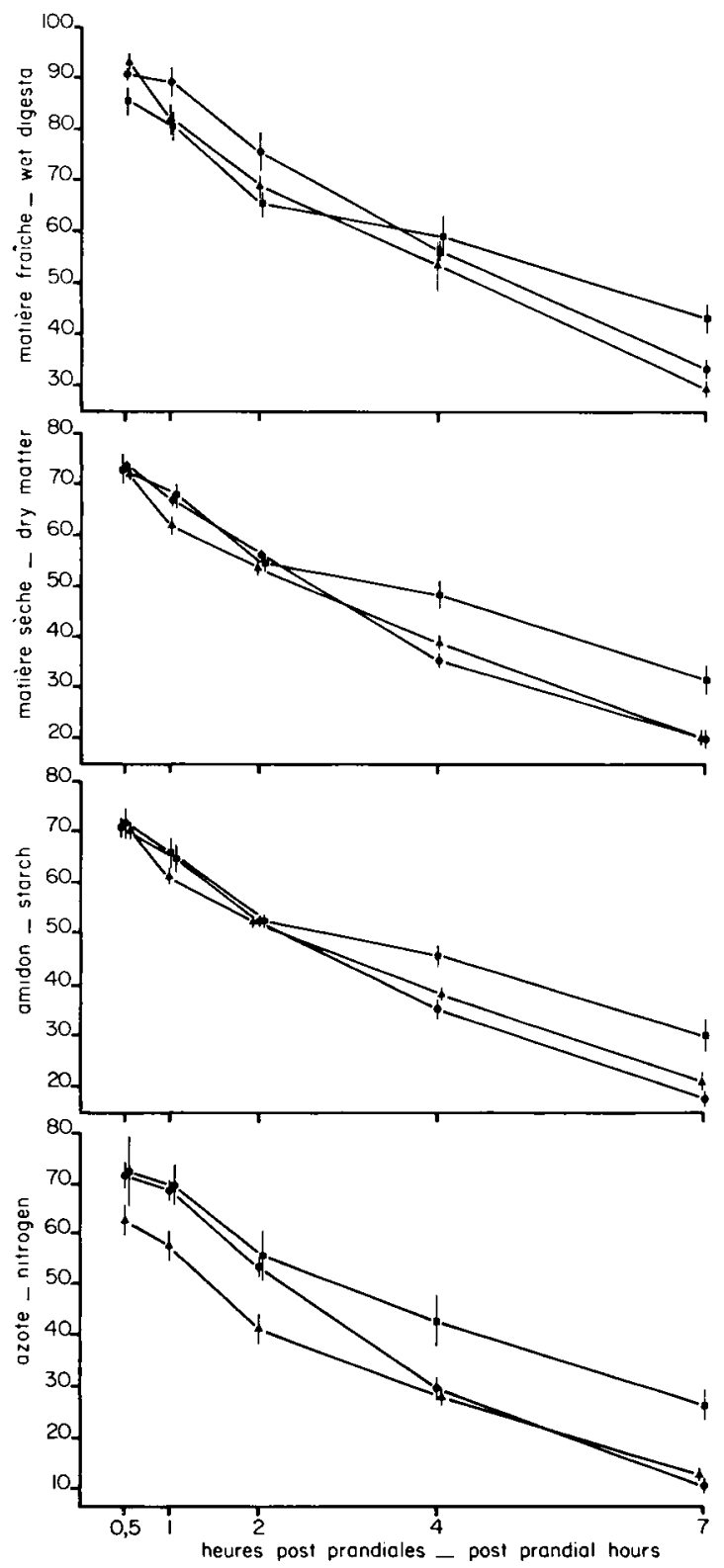

Fig. 1

Quantités moyennes (et écarts-types des moyennes) résiduelles dans l'estomac de matière frấche, de matière sèche, d'amidon et d'azote, exprimées en p. 100 de la quantité ingérée correspondante (tabl. 1), selon la nature de la source protéique associée à l'amidon de blé purifié.

Mean ( $\pm S E M)$ gastric remnants of wet digesta, dry matter, starch and nitrogen, expressed as per cent of the corresponding intake (table 1), according to the protein source associated with purified wheat starch.

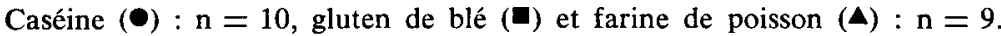
Casein (๑): $n=10$, wheat gluten () and fish meal $(\mathbf{\Delta}): n=9$. 
résiduelles de matière sèche sont très significativement plus faibles que pour l'aliment gluten aux temps $4 \mathrm{~h}$ (caséine et poisson, $\mathbf{P}<0,001$ ) et $7 \mathrm{~h}$ (caséine $\mathrm{P}<0,01$, et poisson $\mathbf{P}<0,001$ ).

Les quantités résiduelles d'amidon sont toujours similaires pour les aliments caséine et poisson : elles sont significativement plus faibles que pour l'aliment gluten aussi bien dans le cas de l'aliment caséine aux temps 4 et $7 \mathrm{~h}(\mathrm{P}<0,01)$ que dans le cas de l'aliment poisson ( $\mathrm{P}<0,01$ à $4 \mathrm{~h} ; \mathrm{P}<0,05$ à $7 \mathrm{~h}$ ). En ce qui concerne enfin l'azote, les quantités résiduelles mesurées pour l'aliment caséine sont, durant les 2 premières heures postprandiales, analogues à celles enregistrées pour l'aliment gluten, mais supérieures à celles obtenues pour l'aliment poisson $(\mathrm{P}<0,01)$; puis à l'inverse, elles sont analogues aux valeurs obtenues pour l'aliment poisson mais très inférieures à celles enregistrées pour l'aliment gluten aux temps $4 \mathrm{~h}(\mathrm{P}<0,05)$ et $7 \mathrm{~h}(\mathrm{P}<0,001)$.

\section{B. Analyse de variance multivariate}

Dans le cas des variables matière fraîche, matière sèche, et amidon, seul l'effet propre de la semaine (terme $\mathrm{c}_{\mathrm{s}}$ ) est significatif, avec des valeurs de la statistique respectivement de 15,40 - 10,35 et 14,66 (rejet de la nullité de l'effet pour les valeurs supérieures à 5,99 avec 1 et 6 degrés de liberté). Toutefois, l'effet global du facteur semaine et du terme d'interaction $\left(c_{s}+d_{r s}\right)$ n'est pas significatif, pour ces trois variables.

En ce qui concerne l'azote (tabl. 3), l'effet global de la semaine inclut une influence significative des deux termes $c_{\mathrm{s}}$ et $d_{r s}$. Par contre, l'effet global du régime $\left(b_{\mathrm{r}}+I_{\mathrm{rp}}\right)$ apparâ̂t lié principalement à l'influence significative du terme d'interaction $\mathrm{I}_{\mathrm{rP}}$.

\section{Tableau 3}

Facteurs de variation de l'évacuation gastrique de l'azote.

La région de rejet de l'hypothèse de nullité de l'effet considéré (terme du modèle d'analyse de variance) est indiquée au seuil de 5 p. 100 selon une statistique qui suit une loi de Wilks.

Sources of variation of gastric emptying of nitrogen.

The rejection of the hypothesis of nullity of the considered effect (term in the model of variance analysis)

is given at the level of 5 p. 100 according to a statistics following the Wilk's law.

\begin{tabular}{c|c|c|c|c}
\hline $\begin{array}{c}\text { Terme } \\
\text { étudié } \\
\begin{array}{c}\text { Studied } \\
\text { term }\end{array}\end{array}$ & $\begin{array}{c}\text { Degrés } \\
\text { de liberté } \\
\text { Degrees } \\
\text { of freedom }\end{array}$ & $\begin{array}{c}\text { Rejet } \\
\text { de la nullité } \\
\text { Rejection } \\
\text { of nullity }\end{array}$ & $\begin{array}{c}\text { Valeurs } \\
\text { de la statistique } \\
\text { Values } \\
\text { of the statistics }\end{array}$ & $\begin{array}{c}\text { Effets } \\
\text { significatifs } \\
\text { Significant } \\
\text { effects }\end{array}$ \\
\hline $\mathrm{b}_{\mathrm{r}}$ & 2,6 & $>22,13$ & 19,68 & - \\
$\mathrm{I}_{\mathrm{rp}}$ & 6,6 & $>54,49$ & $\rightarrow \infty$ & $*$ \\
$\mathrm{c}_{\mathrm{s}}$ & 1,6 & $>5,99$ & 37,44 & $* *$ \\
$\mathrm{~d}_{\mathrm{rs}}$ & 2,6 & $>22,13$ & 26,58 & $* *$ \\
$\mathrm{~b}_{\mathrm{r}}+\mathrm{I}_{\mathrm{rp}}$ & 6,8 & $>71,37$ & $\rightarrow \infty$ & $* *$ \\
$\mathrm{c}_{\mathrm{s}}+\mathrm{d}_{\mathrm{rs}}$ & 3,6 & $>25,87$ & 35,70 & $* *$ \\
\hline
\end{tabular}



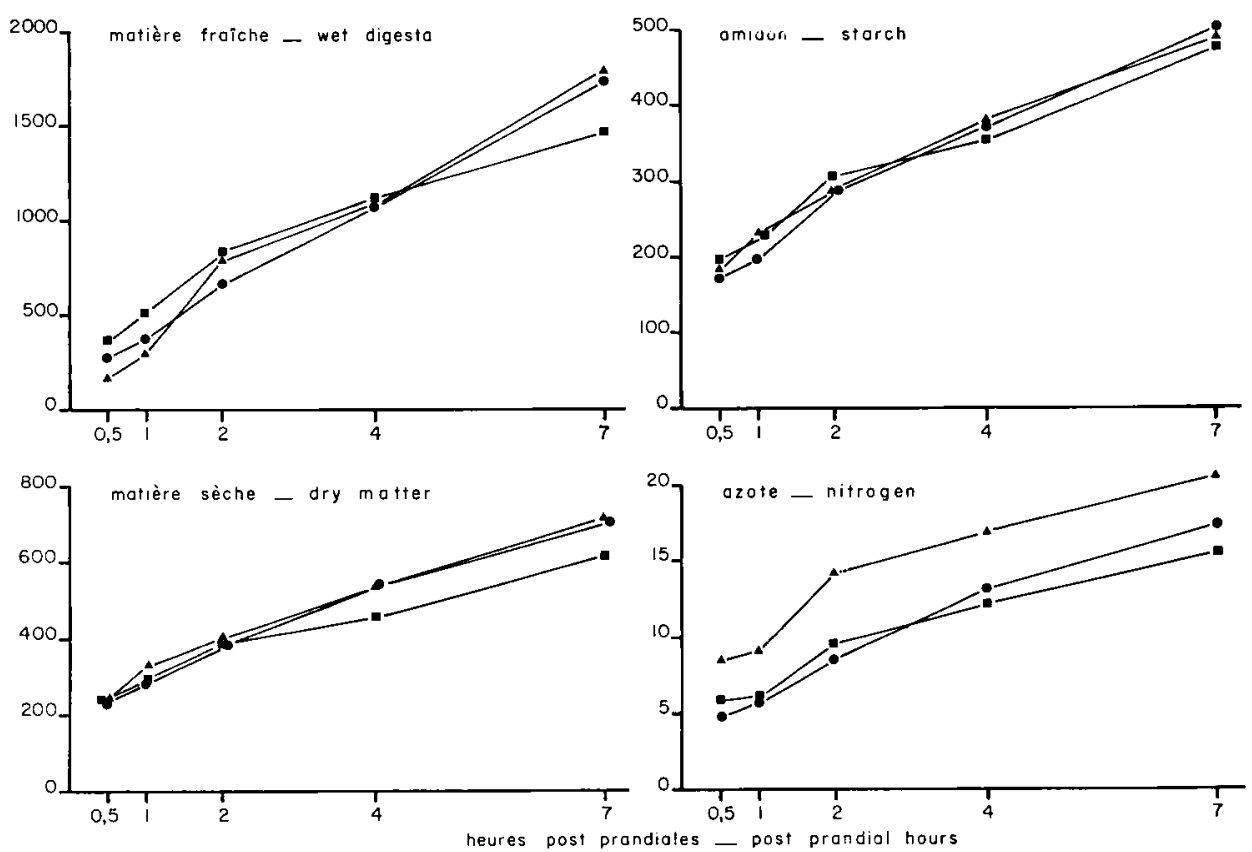

FIG. 2

Courhes moyennes d'évacuation gastrique (quantités évacuées en g) résultant de l'estimation du seul terme $b_{r}$ qui exprime l'effet fixe du régime dans le modèle d'analyse de variance.

Mean gastric emptying curves (emptied quantities as $g$ )

resulting from the estimation of the term $b_{r}$ alone,

representative of the fixed effect of the diet in the model used for variance analysis.

Symboles : voir fig. 1. Chaque point calculé correspond à 6 mesures.

Symbols : see fig. 1. Each calculated point is representative of 6 measurements.

En définitive, et pour le nombre relativement restreint de données (18 vecteurs) disponibles pour l'analyse multivariate, l'influence du régime en lui-même hors de toute influence aléatoire du porc, éest-à-dire l'effet de la protéine associée à un même amidon, n'est jamais significative quelle que soit la variable considérée. On peut procéder à l'estimation du terme $b_{r}$ de façon à construire les courbes d'évacuation exprimant le seul effet fixe du régime (fig. 2). Mais l'absence d'influence significative de ce dernier fait qu'il n'est pas justifié de procéder à un ajustement en fonction du temps par le modèle de PotThoff \& Roy pour apprécier d'éventuelles différences de la cinétique du phénomène au travers de tests de parallélisme. 


\section{Discussion}

Le seul examen de la décroissance des quantités résiduelles dans l'estomac au cours des premières heures postprandiales ne permet aucune distinction claire entre les trois aliments mis en comparaison, en dépit de quelques différences ponctuelles selon les tests deux à deux de ces quantités moyennes. Par contre, les bilans à la fin de la quatrième et de la septième heures postprandiales montrent que, quelle que soit la variable contrôlée, les quantités résiduelles dans l'estomac sont analogues pour les régimes caséine et poisson, mais qu'elles sont significativement plus importantes pour le régime gluten. Ceci implique que l'évacuation gastrique de ce dernier est au total plus lente en $7 \mathrm{~h}$ que celle du régime poisson. Cette observation coïncide avec celle de Zebrowska (1973 a) selon laquelle l'évacuation du régime gluten est aussi plus lente globalement (en $8 \mathrm{~h}$ ) que celle de régimes soja ou féverole. Par contre, selon ce même auteur, l'évacuation gastrique d'un régime caséine est aussi lente que celle du régime gluten; or, selon nos bilans des quantités résiduelles à $7 \mathrm{~h}$, elle apparaît notablement plus rapide que celle du régime gluten et même aussi rapide que celle du régime poisson. Cette apparente contradiction doit être analysée avec prudence dans la mesure où de tels bilans peuvent être relativement trompeurs.

Dans une comparaison de cet ordre entre régimes, un bilan d'évacuation à tel ou tel temps ne rend pas compte du décours de l'évacuation c'est-à-dire de la cinétique du phénomène qui conduit au bilan final. Cet aspect cinétique est apparu comme étroitement influencé par la source de protéines alimentaires (Laplace, Pons \& Simon, 1983), mais il n'est pas possible de le prendre ici en compte valablement en l'absence d'effet régime significatif pour les données utilisées dans l'analyse. Par ailleurs, un bilan d'évacuation au terme d'un laps de temps donné est la résultante d'un niveau moyen d'évacuation qui s'avère essentiellement influencé par la nature de l'association d'un amidon et d'une protéine dans un régime (Laplace, Pons \& Simon, 1983). Ceci signifie que le désaccord sur les bilans respectifs pour les régimes gluten et caséine, similaires selon ZEBRowsKa (1973 a) et différents selon nos mesures, pourrait fort bien ne tenir qu'à la différence de nature des constituants amylacés associés au gluten ou à la caséine. Alors que l'aliment ici utilisé ne comporte que de l'amidon de blé, il contient dans l'expérience de Zebrowska (1973 a) outre lamidon de blé, de la pomme de terre, du glucose et du saccharose.

D'autres aspects d'ordre méthodologique peuvent également interférer dans l'évaluation des effets du régime, et au premier chef l'influence des animaux eux-mêmes. Son importance a déjà été soulignée dans la mesure où l'effet de l'animal peut conduire à une translation des courbes d'évacuation et partant à fausser l'évaluation du niveau moyen (donc des bilans) d'évacuation gastrique (LAplace, Pons \& Simon, 1983), d'autant plus que le nombre de porcs testés est faible. Le risque est bien réel dans notre expérience où des porcs différents sont affectés à chacun des régimes et où l'effet du terme hiérarchique $I_{r p}$ s'avère significatif pour la matière sèche et l'azote. L'erreur potentielle n'est pas moins grande dans le travail de ZebrowsKA (1973 a) où, selon les cas, les porcs ont reçu un seul ou plusieurs des régimes comparés, l'évaluation de l'évacuation gastrique étant de surcroît fondée sur l'usage de canules duodénales placées entre 30 et $80 \mathrm{~cm}$ en aval du pylore. Enfin, un dernier facteur parasite est constitué par l'effet, significatif pour toutes les variables étudiées, du terme $c_{s}$. Ceci revient 
à dire que les deux cinétiques constituées consécutivement ne sont pas d'authentiques répétitions et que le nombre d'essais réalisés chez chaque porc peut avoir une influence du même ordre que celle du nombre de porcs utilisés.

L'examen des courbes exprimant l'effet fixe du régime (fig. 2) conduit à noter, pour la matière fraîche, la matière sèche et l'amidon, une très grande similitude avec les profils de la figure 1, compte tenu des modes d'expression et unités employés dans chaque cas. De plus, on remarque, à défaut de comparaison rigoureuse des profils (fonction du temps), que l'évacuation gastrique est toujours la plus régulière (pratiquement linéaire) pour le régime caséine. Le bilan au terme de la septième heure postprandiale confirme, pour la matière fraîche et la matière sèche, une similitude pour les régimes caséine et poisson et l'évacuation plus lente du régime gluten. Le cas de la matière fraîche a peu d'intérêt en lui-même dans la mesure où les volumes d'eau sécrétoire ajoutée aux quantités résiduelles dans l'estomac ne sont pas contrôlés. En ce qui concerne la matière sèche, la moindre évacuation gastrique du régime gluten à 4 et $7 \mathrm{~h}$ après le repas, par rapport aux valeurs enregistrées pour les régimes caséine et poisson, correspond à un retard important (équivalant à 10 p. 100 de la quantité de matière sèche ingérée). L'ampleur de ce phénomène est suffisante pour que l'on puisse valablement supposer qu'il recouvre un phénomène physiologique, même s'il n'est pas significatif selon l'analyse multivariate, et ce probablement en raison de la limitation du nombre des données (Laplace, Pons \& Simon, 1983). Dans le cas de l'amidon, l'absence d'effet du régime pourrait surprendre compte tenu des résultats antérieurs : en effet, sur la base de l'évacuation de 4 aliments, dont les régimes gluten et poisson ici pris en compte, il a été précédemment mis en évidence par analyse univariate des effets significatifs de la source de protéines et du couple amidon-protéine à l'égard de l'évacuation de l'amidon (Laplace, Pons \& Simon, 1983). Même si le nombre restreint de données ici utilisées n'est pas étranger à la non-signification de l'effet, il semble aussi que les divergences entre régimes du fait de la source de protéines soient plus grandes lors d'association à un amidon de maïs (LAPlace, Pons \& Simon, 1983) que lors d'association à un amidon de blé comme c'est ici le cas.

En ce qui concerne enfin l'évacuation de l'azote, on retrouve un résultat identique à celui du travail précédent (LAPlace, Pons \& Simon, 1983) : au total l'effet du régime ne peut être considéré comme nul mais il n'est pas, en tant qu'effet propre, identifié comme significatif dans la mesure où il est en grande partie masqué par l'effet porc. Ceci étant, il existe une différence sensible à 4 et $7 \mathrm{~h}$ des valeurs obtenues pour le régime caséine par rapport aux deux autres régimes, selon que l'on considère les quantités résiduelles (fig. 1) ou les quantités évacuées (fig. 2). Mais dans ce dernier cas, et pour le régime caséine, les valeurs correspondent à 6 mesures sur 3 porcs au lieu de 10 sur 5 porcs. Néanmoins, entre $30 \mathrm{mn}$ et $2 \mathrm{~h}$ après le repas, l'écart considérable entre la courbe d'évacuation du régime poisson et les 2 autres courbes recouvre assurément une différence réelle, quoique non révélée par l'analyse de variance comme un effet significatif. Cette différence est vraisemblablement liée à l'évacuation gastrique rapide et précoce de l'importante fraction soluble des protéines de poisson : 31,6 p. 100 de l'azote total de l'aliment contre 2,3 et 1,2 p. 100 dans le cas du gluten et de la caséine respectivement.

Si cet écart des courbes d'évacuation de l'azote peut être aisément expliqué, celui qui est enregistré pour l'évacuation de la matière sèche entre régimes caséine et poisson d'une part et gluten d'autre part, est moins facile à relier à une cause simple. L'existence d'une telle différence implique l'intervention, variable selon le régime, de 
certains des facteurs de contrôle du processus d'évacuation gastrique. Aucune information particulière ne permet de mettre en cause des facteurs physiques tels que d'éventuelles modifications de la viscosité ou du pH des contenus gastriques. Les différences ne peuvent pas non plus être expliquées par la légère différence du taux d'amidon (moindre dans le régime poisson) ni par la présence de 2 p. 100 d'huile dans le seul régime caséine, ni par les faibles écarts de teneur réelle en azote des régimes, ni par leurs différences de concentration en énergie (tabl. 1). En fait, la différence essentielle entre les 3 régimes est bien liée à la source de protéines, donc aux conséquences potentielles des différences de leurs vitesses d'hydrolyse respectives et des proportions relatives de leurs acides aminés constitutifs.

L'influence, sur l'évacuation gastrique, des acides aminés libérés au terme de l'hydrolyse des protéines, a fait l'objet de diverses évaluations. Pour STEPhens, Woolsov \& CoOKe (1975), le tryptophane, seul de tous les acides aminés, ralentit l'évacuation gastrique chez le chien; cet effet résulterait de la mise en jeu de récepteurs au niveau de la paroi duodénale et jéjunale (COOKE \& WARD, 1976 ; CoOKE, 1977) plutôt que d'une libération de cholécystokinine (STEPHENS, WOOLSON \& COOKE, 1975). L'effet inhibiteur des solutions d'acides aminés a été relié chez l'homme à leur osmolarité (Cooke \& Moulang, 1972 ; Burn-Murdoch, Fisher \& Hunt, 1978) voire à leur effet d'acide faible (Fisher \& HuNT, 1977). Enfin, chez le porc, Ochia (1976) n'a observé aucune modification du rythme d'évacuation sous l'influence de la glycine, de la lysine, du tryptophane, de la méthionine, ou de l'acide glutamique. Mais tous ces travaux concernent l'évacuation de «repas d'épreuve» liquides, situation a priori peu favorable pour saisir une influence qui s'exercerait sur le mécanisme responsable de l'évacuation des matériaux alimentaires.

Dans le cas des régimes dont l'évacuation gastrique est ici comparée, lexamen du tableau 2 conduit à noter que le rapport des acides aminés essentiels/non essentiels est de 1,10 pour le poisson et 1,06 pour la caséine contre 0,60 seulement pour le gluten. Par ailleurs, on peut souligner qu'il y a environ 5 fois plus de lysine, 3 à 4 fois moins de cystine, 1,8 fois plus de méthionine et 2,5 à 3 fois plus d'acide aspartique + asparagine dans les régimes caséine et poisson que dans le régime gluten. Ces observations sont convergentes avec le fait que l'évacuation de la matière sèche est analogue pour caséine et poisson, mais différente i.e. plus lente pour le gluten. Il est donc plausible qu'une influence complexe de la composition en acides aminés des protéines alimentaires soit responsable de la similitude vs. différence d'évacuation gastrique de la matière sèche. Par contre, l'hypothèse d'un rôle de la proline, telle qu'envisagée au début de ce mémoire, ne paraît pas fondée dans la mesure où les régimes caséine et gluten apportent 2,7 à 2,8 fois plus de proline que le régime poisson. Ce cas particulier mis à part, la question se trouve donc posée du rôle exact des acides aminés dans ceux des mécanismes de contrôle de l'évacuation gastrique qui reposent sur une intéroception prenant origine au niveau de l'intestin grêle (MEI, 1981). On sait en effet qu'existent, au niveau de la muqueuse intestinale, des chémorécepteurs vagaux mis en jeu de façon plus ou moins spécifique par un ou plusieurs acides aminés (JEanningros \& MeI, 1980). Mais l'analyse précise de ces effets requiert une méthodologie adaptée prenant aussi en compte de façon très précise la nature des glucides du régime dans la mesure où les récepteurs vagaux concernés sont polymodaux et où une interaction pourrait s'exercer à ce niveau. 


\section{Conclusion}

L'influence de la source de protéines alimentaires à l'égard de l'évacuation gastrique d'un régime à base d'amidon de blé peut se traduire de deux façons. Une forte proportion d'azote soluble conduit à enregistrer une évacuation gastrique de lazote total plus importante (cas du poisson par rapport à la caséine et au gluten). Par ailleurs, l'évacuation gastrique de la matière sèche s'avère plus lente pour le régime gluten que pour les régimes caséine et poisson. La similitude d'évacuation pour ces derniers est à rapprocher de leur analogie de composition en acides aminés, alors que la composition fort différente dans le cas du régime gluten pourrait répondre de l'évacuation plus lente de ce dernier.

\section{Summary}

Gastric emptying and amino acid composition of the dietary proteins in the pig

Five Large White castrated male pigs weighing $52.5 \mathrm{~kg}$ on an average were fitted with permanent gastric cannulas to measure the residual gastric contents of wet digesta, dry matter, starch and nitrogen at various times after a test meal (30 min, 1, 2, 4 and $7 \mathrm{hrs}$ ). The diet was based on a purified wheat starch and the protein source was casein hydrochloric. Two measurements were performed on separate days for each collection time in each pig. These results were compared with those previously recorded (LAPLACE et al., 1981) using identical methods to test diets based on the same purified wheat starch but including either wheat gluten or fish meal as protein source. The respective amino acid composition of the three diets was determined (table 2).

The picture given by the decreasing residual quantities collected from the stomach (fig. 1) showed a strong similarity for the casein and fish meal diets, while the residual quantities were significantly higher 4 and $7 \mathrm{hrs}$ after the wheat gluten diet. These dietinduced differences were not significant according to a multivariate analysis of the quantities emptied from the stomach, while significant intercurrent effects of time and pigs occurred. However, the estimation of the fixed effect of the diet allowed to calculate curves of gastric emptying (fig. 2) leading to grossly similar conclusions as compared to those resulting from figure 1 . It thus appeared that the dietary protein source led to 2 kinds of effects : (i) Gastric emptying of total nitrogen was influenced by the proportion of soluble nitrogen in the diet, mainly during the early postprandial period : emptying of total nitrogen was much higher for the fish meal diet (31.6 per cent soluble) than for the casein or gluten diets ( 1.2 or 2.3 per cent), (ii) The gastric emptying of dry matter was closely similar for the casein and fish meal diets whose amino acid compositions (table 2) also showed numerous similarities. Opposite to that, emptying of dry matter was slower for the wheat gluten diet, the amino acid composition of which was very different.

Key words : Gastric emptying, pig, amino acids, digestion, dietary proteins.

Reçu en octobre 1981. 


\section{Références bibliographiques}

Burn-Murdoch R.A., Fisher M.A., Hunt J.N., 1978. The slowing of gastric emptying by proteins in test meals. $J$. Physiol. (London), 274, 477-485.

Cooke A.R., 1977. Localization of receptors inhibiting gastric emptying in the gut. Gastroenterology, 72, 875-880.

Cooke A.R., Moulang J., 1972. Control of gastric emptying by aminoacids. Gastroenterology, 62, 528-532.

COOKE A.R., WARD W.O., 1976. Effect of tryptophan and its metabolites on gastric emptying of liquid meals in dogs. Proc. Soc. Exp. Biol. Med., 152, 656-658.

Cuber J.C., Laplace J.P., 1979. Evacuation gastrique de l'amidon et de l'azote d'un régime semi-purifié à base d'amidon de maïs chez le porc. Ann. Zootech., 28, 173-184.

Cuber J.C., Laplace J.P., Villiers P.A., 1980. Fistulation de l'estomac et contenus gastriques résiduels après ingestion d'un régime semi-purifié à base d'amidon de maïs chez le porc. Reprod. Nutr. Develop., 20, 1161-1172.

Fisher M.A., HunT J.N., 1977. Effects of hydrochlorides of aminoacids in test meals on gastric emptying. Digestion, 16, 18-22.

Henry Y., Pion R., Rerat A., 1976. Protein supply for pigs and possibilities of reducing protein feeding standards. World Rev. Anim. Prod., 12, 9-32.

Jeanningros R., Mei N., 1980. Données préliminaires sur la réponse des chémorécepteurs intestinaux aux acides aminés. Reprod. Nutr. Develop., 20, 1615-1619.

Laplace J.P., Pons O., Cuber J.C., Kabore Ch., Villiers P.A., 1981. Effets de la nature de l'amidon (blé ou maïs) et des protéines (poisson ou gluten) sur les facteurs de contrôle et le décours de l'évacuation gastrique d'un régime semi-purifié chez le porc. Applications de l'analyse multidimensionnelle et de la régression polynomiale. Ann. Zootech., 30, 209-248.

Laplace J.P., Pons O., Simon P., 1983. Révision des effets de l'amidon et des protéines sur l'évacuation gastrique chez le porc. Ann. Zootech., 32, 507-524.

Mer N., 1981. La sensibilité viscérale. J. Physiol., Paris, 77, 597-612.

OCHIA B.A., 1976. Influence of aminoacids on gastric emptying in young pigs. Acta Physiol. Acad. Sci. Hung., 48, 50-51.

Pion R., Fauconneau G., 1966. Les acides aminés des protéines alimentaires. Méthodes de dosage et résultats obtenus. Cahier $n^{\circ} 6$ : Aminoacides, peptides, protéines, 157-175, A.E.C., Commentry.

PotthofF R.F., Roy S.N., 1964. A generalized multivariate analysis of variance model useful especially for growth curve problems. Biometrika, 51, 313-326.

Rerat A., Lougnon J., 1963. Etudes sur le transit digestif chez le Porc. Ann. Biol. Anim. Biochim. Biophys., 3 ( $\mathrm{N}^{\prime \prime}$ hors sérite), 21-27.

STEPHENS J.R., Woolson R.F., CoOKe A.R., 1975. Effects of essential and nonessential aminoacids on gastric emptying in the dog. Gastroenterology, 69, 920-927.

Zebrowska T., 1973 a. Influence of dietary protein source on the rate of digestion in the small intestine of pigs. 1) Amount and composition of digesta. Rocz. Nauk. Roln., $95 \mathrm{~B}, 115-133$.

ZeBRowsKA T., $1973 \mathrm{~b}$. Influence of dietary protein source on the rate of digestion in the small intestine of pigs. 2) The rate of protein digestion and amino acids absorption. Rocz. Nauk. Roln., 95 B, 135-155.

ZEBrowSKa T., BURACZEWSKa L., 1972. Influence of dietary protein level on the rate of digestion in the small intestine of pigs. 1) Amount and composition of digesta (en polonais). Rocz. Nauk. Roln., 94 B, 81-95.

Zebrowska T., Buraczewska L., Buraczewski S., Horszczaruk F., 1975. Digestion and absorption in the small intestine of pigs. 1) Digestion and absorption of dry matter and nitrogen. Rocz. Nauk. Roln., 96 B, 79-90. 